\title{
The roles of serum alkaline and bone alkaline phosphatase levels in predicting heterotopic ossification following spinal cord injury
}

\author{
M Citak ${ }^{1}$, D Grasmücke ${ }^{1}$, EM Suero ${ }^{2}$, O Cruciger ${ }^{3}$, R Meindl $^{1}$, TA Schildhauer ${ }^{3}$ and M Aach ${ }^{1}$
}

\begin{abstract}
Study design: Retrospective chart review.
Objectives: To analyze the usefulness of serum alkaline phosphatase (AP) and bone alkaline phosphatase (BAP), as well as C-reactive protein (CRP) levels in predicting heterotopic ossification (HO).

Setting: Department of Spinal Cord Injury and Department of General and Trauma Surgery, BG-University Hospital Bergmannsheil, Ruhr University Bochum, Germany.

Methods: Between January 2003 and December 2013, 87 patients with HO around the hips met the inclusion criteria and were included in the study. Alkaline phosphatase, CRP and BAP were assessed and interpreted the time of HO diagnosis and after radiation therapy in all patients.

Results: At the time of $\mathrm{HO}$ diagnosis, 49 out of 87 patients (49.4\%) had elevated alkaline phosphatase levels and 39 out of 87 patients (44.8\%) had elevated BAP levels. Elevated CRP values were found in 67 patients (77.0\%). Within 3 days after single-dose radiation therapy, elevated AP levels persisted in 38 patients (43.7\%) and elevated BAP levels in 28 patients (32.2\%).

Conclusions: The results obtained show that the determination of CRP, AP and BAP levels may not be considered a reliable screening method for early $\mathrm{HO}$ detection, subsequent to spinal cord injury.
\end{abstract}

Spinal Cord (2016) 54, 368-370; doi:10.1038/sc.2015.211; published online 8 December 2015

\section{INTRODUCTION}

A high incidence of heterotopic ossification $(\mathrm{HO})$ subsequent to traumatic spinal cord injury (SCI) has been reported in previous studies. ${ }^{1,2}$ According to these, early detection and adequate treatment of $\mathrm{HO}$ is essential to avoid ankylosis. Despite the high incidence, only little evidence exists regarding the onset of HO in SCI patients. Furthermore, despite the usefulness of laboratory screening parameters as a tool for early detection of neurogenic HO, this has not been fully elucidated.

Larson et al. ${ }^{3}$ reported on the increased prevalence of HLA-B27 in patients with ectopic ossification following traumatic SCI. In contrast to this study, Garland et al. ${ }^{4}$ concluded that there was no positive correlation between the human leukocyte antigen (HLA) antigen system and $\mathrm{HO}$ onset subsequent to brain trauma or SCI. Schurch et al. ${ }^{5}$ found abnormal 24-h prostaglandin E2 (PGE2) excretion levels in SCI patients with HO for whom the bone scan had not yet stabilized. More recently, Estrores et al. ${ }^{6}$ analyzed the serum levels of C-reactive protein (CRP) and erythrocyte sedimentation rate (ESR) among $\mathrm{HO}$ patients. On the basis of this retrospective study including patients with $\mathrm{HO}$, they postulated that CRP is a more specific parameter than ESR for monitoring the activity of HO after SCI. Singh et al. ${ }^{7}$ reported about a correlation of elevated serum levels of creatine phosphokinase in predicting HO in SCI patients. Sherman et al. ${ }^{8}$ postulated that elevated serum creatine kinase may be associated with a more severe course of $\mathrm{HO}$ and a higher insensitivity to the etidronate therapy. However, to the best of our knowledge, there exists no reliable evidence in the literature for laboratory screening methods aimed at predicting $\mathrm{HO}$ in SCI patients. Nevertheless, the use of bone alkaline phosphatase (BAP) has proven to be a sensitive and reliable indicator of bone metabolism and therefore is a useful tool in the diagnosis and assessment of metabolic bone diseases. ${ }^{9-11}$

The main objective of the current study was to analyze the importance of BAP in predicting $\mathrm{HO}$ in SCI patients. The second aim of the study was to investigate the usefulness of alkaline phosphatase or CRP in predicting HO.

\section{MATERIALS AND METHODS}

The study was conducted after obtaining approval from our Institutional Review Board. All SCI patients treated between January 2003 and December 2013, who subsequently developed HO around the hips, were enrolled in this study. Patients under 18 years of age and those without sufficient record of alkaline phosphatase, BAP and CRP at the time of HO diagnosis were excluded from the final analysis. Patients with the following bone diseases, which may influence especially the BAP values, were also excluded from the study: Paget's disease, osteosarcoma, malignant tumors, bone metastasis, renal osteodystrophy, multiple myeloma, vitamin D deficiency, primary and secondary hyperparathyroidism. A total of 259 patients developed $\mathrm{HO}$ around the hip

${ }^{1}$ Department of Spinal Cord Injury, BG-University Hospital Bergmannsheil, Ruhr University Bochum, Bochum, Germany; ${ }^{2}$ Department of Trauma Surgery, Hannover Medical School, Hannover, Germany and ${ }^{3}$ Department of General and Trauma Surgery, BG-University Hospital Bergmannsheil, Ruhr University Bochum, Bochum, Germany Correspondence: Dr M Citak, Department of Spinal Cord Injury, BG-University Hospital Bergmannsheil, Ruhr University Bochum, Bürkle-de-la-Camp Platz 1, Bochum 44789, Germany.

E-mail: mcitak@gmx.de

Received 2 April 2015; revised 28 October 2015; accepted 30 October 2015; published online 8 December 2015 
and were treated in the Department of Spinal Cord Injuries at the BG-University Hospital Bergmannsheil, Bochum, Germany. Eighty-seven out of 259 patients met the inclusion criteria and were enrolled. Diagnosis of HO was accomplished using our standardized hospital protocol. According to the protocol, patients are screened biweekly by ultrasound examinations of the hip and shoulder joints by our experienced radiologists. In case of suspicion of $\mathrm{HO}$, the presumptive clinical diagnosis was confirmed with either computed tomography (CT) or magnetic resonance imaging (MRI).

General information about age, sex, severity of spinal cord lesion according to the American Spinal Injury Association (ASIA) Impairment Scale (AIS), ${ }^{12}$ time interval between the SCI and HO diagnosis (in days) and length of hospital stay was obtained from the patients' medical records. Alkaline phosphatase, CRP and BAP were analyzed at the time of $\mathrm{HO}$ diagnosis and within 3 days after single-dose radiation therapy. Normal levels of serum BAP were defined as $<50 \mathrm{Ul}^{-1}$. Alkaline phosphatase values $<127 \mathrm{Ul}^{-1}$ were classified as normal. Normal value for CRP was $<1.0 \mathrm{mg} \mathrm{dl}^{-1}$.

\section{Statistical analysis}

Descriptive statistics are presented in the form of number of occurrences and percentage, or mean, s.d. and range. The comparison of CRP, BAP and AP levels prior to and after radiation therapy were made using the Mann-Whitney test. Significance level was set to $\alpha=0.05$. All data were processed using a statistical analysis software (GraphPad Prism version 5.0d, La Jolla, CA, USA).

\section{RESULTS}

Eighty-seven SCI patients with HO around the hips were enrolled in this retrospective study. The patient cohort consists of 38 paraplegic (43.7\%) and 49 tetraplegic patients (56.3\%) with a mean age of 42.3 years $(18-76$ years; s.d.=17.9). Seventy-nine patients were male $(90.8 \%)$ and eight patients were female (9.2\%). Seventy-nine out of

Table 1 Mean CRP, AP and BAP levels over time after initial SCI development

\begin{tabular}{lcccc}
\hline HO development & $N$ & Mean AP & Mean BAP & Mean CRP \\
\hline Within 14 days & 1 & 208 & 61 & 2 \\
15-29 days & 7 & 117.3 & 50.6 & 4 \\
30-49 days & 25 & 269.3 & 92.6 & 5.9 \\
50-69 days & 20 & 294.6 & 92.1 & 5 \\
70-89 days & 16 & 111.5 & 50.6 & 2.6 \\
90-99 days & 3 & 151.5 & 61.3 & 2.6 \\
$>100$ days & 15 & 103.1 & 49.6 & 4.2 \\
\hline
\end{tabular}

87 patients (90.8\%) had a complete lesion according to the AIS A; one patient (1.2\%) had an AIS B lesion; five patients (5.7\%) had an AIS C lesion; and one patient $(1.2 \%)$ had an AIS D lesion. The mean time interval between the initial trauma and the diagnosis of $\mathrm{HO}$ was 68.1 days \pm 42.4 (range, $13-295$ days). In the majority of the cases, HO affected both the hips (74.7\%). In the remaining 22 cases $(25.3 \%)$, HO occurred unilaterally, with the left side being more commonly affected $(N=13)$.

Analyzing laboratory parameters at the time of $\mathrm{HO}$ diagnosis, 49 out of 87 patients $(49.4 \%)$ presented elevated levels of alkaline phosphatase and 39 out of 87 patients $(44.8 \%)$ had elevated BAP. Elevated CRP was found in 67 patients $(77.0 \%)$. The mean AP was $207.6 \pm 275.1 \mathrm{Ul}^{-1}$ (range, 41-1750 Ul-1), and the mean BAP was $72.5 \pm 95.6 \mathrm{Ul}^{-1}$ (range, 10-750 $\mathrm{Ul}^{-1}$ ). The average CRP was $4.9 \pm 6.7 \mathrm{mg} \mathrm{dl}^{-1}$ (range, $0.1-40.0 \mathrm{mg} \mathrm{dl}^{-1}$ ). The majority of the patients $(N=45$ patients; $51.7 \%)$ developed HO between 30 and 69 days post injury. During this time, mean AP, BAP and CRP levels were significantly higher compared with other onset periods (Table 1, Figure1).

However, it must be noted that, during this period (30-69 days), 23 out of 45 patients $(51.1 \%)$ had normal serum BAP levels and normal AP levels were found in 18 out of 45 patients (40.0\%), as well as normal CRP values in 7 out of the 45 patients (15.6\%). Within 3 days after single-dose radiation therapy ( $7 \mathrm{~Gy}$ ), elevated AP persisted in 38 patients $(43.7 \%)$ and BAP in 28 patients $(32.2 \%)$, although no further signs of progression of $\mathrm{HO}$ were observed. After radiation therapy, the mean AP levels decreased, but not significantly, to $181.6 \pm 259.4 \mathrm{Ul}^{-1}$ (range, 35-1652 $\left.\mathrm{Ul}^{-1}\right)(P=0.48)$. The average BAP post radiation was $44.8 \pm 34.3 \mathrm{Ul}^{-1}$ (range, $\left.10-160 \mathrm{Ul}^{-1}\right)(P=0.06)$.

Significant pre-post differences were observed for CRP values after radiation therapy with a mean of $2.5 \pm 3.3 \mathrm{mg} \mathrm{dl}^{-1}$ (range, $\left.0.2-21 \mathrm{mg} \mathrm{dl}^{-1}\right)(P=0.002)$.

\section{DISCUSSION}

To the authors' best knowledge, this is the largest retrospective clinical study analyzing the role of laboratory parameters in predicting/ monitoring $\mathrm{HO}$ due to SCI. Estrores et al. ${ }^{6}$ analyzed a cohort of 37 patients focusing on the importance of CRP and ESR for monitoring the inflammatory activity of HO after SCI. In this study, ESR and CRP were elevated in the acute phase of $\mathrm{HO}$ in all patients. However, in the current study, only 67 patients (77\%) had elevated levels of CRP at the time of $\mathrm{HO}$ diagnosis.

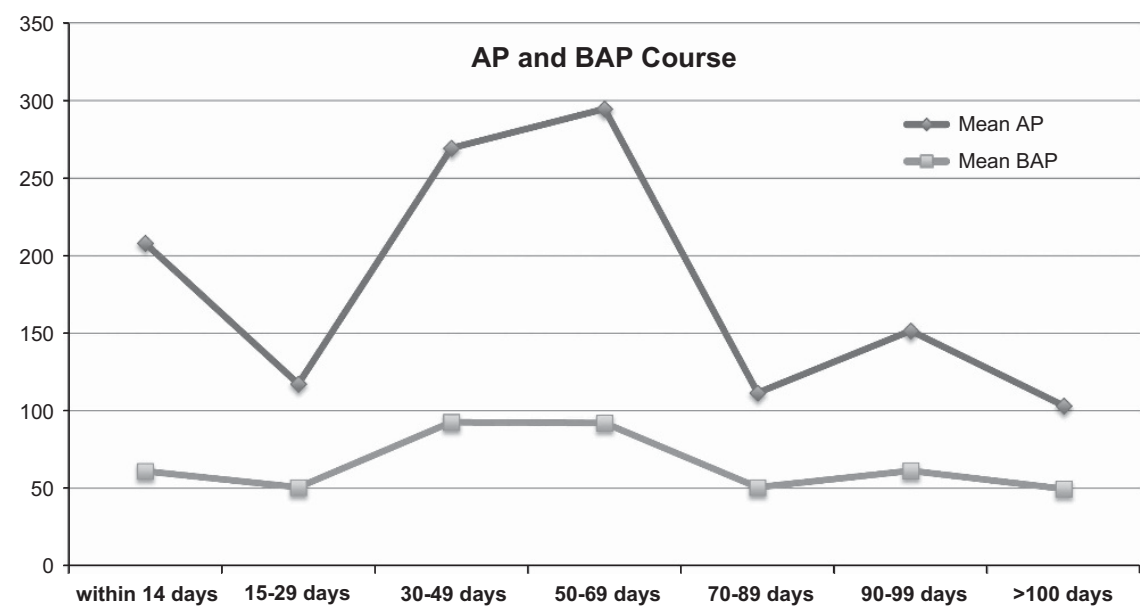

Figure 1 Mean AP and mean BAP values. Higher levels for BAP and AP were seen between 30 and 69 after initial SCl. A full color version of this figure is available at the Spinal Cord journal online. 
Orzel and Rudd ${ }^{13}$ concluded that serum levels of alkaline phosphatase can be used to detect early onset of $\mathrm{HO}$ in a series of 50 patients. In opposition to those findings, Kedlaya and Nazir ${ }^{14}$ reported on 3 cases with immature $\mathrm{HO}$ and normal serum alkaline phosphatase (AP) levels and questioned the benefits of AP as a screening parameter in predicting $\mathrm{HO}$. The results obtained through our study support the conclusion of Kedlaya and Nazir, as only $49.4 \%$ of cases had elevated AP levels at the time of $\mathrm{HO}$ diagnosis.

Previous studies have concluded that BAP is a reliable method for the diagnosis and assessment of metabolic bone diseases. However, in our series, only $44.8 \%$ of the patients had elevated BAP levels at the time of $\mathrm{HO}$ diagnosis. Our study provides valuable, new and important information to the literature. One interesting finding is that the majority of $\mathrm{HO}$ cases were diagnosed between 30 and 69 days after spinal cord injury. In this time period, the mean AP, BAP and CRP levels were higher compared with other time periods of $\mathrm{HO}$ onset. However, during this period, high rates of normal AP and BAP levels were also noted. Therefore, the determination of AP, BAP and $\mathrm{CRP}$ values may not be considered a reliable method in predicting $\mathrm{HO}$, as only half of the cases could be detected using those specific laboratory parameters. One of the strengths of our study is the early screening for HO using ultrasound in all patients with SCI, followed by further diagnostics to confirm HO via CT or MRI. Furthermore, this study investigated the results of a large number of patients with $\mathrm{HO}$ and SCI.

Traditionally radiotherapy has been used in our facility for prophylaxis and treatment of neurogenic HO. Schurch et al. ${ }^{5}$ demonstrated a marked increase in PGE2 levels in SCI patients with $\mathrm{HO}$ that had not yet reached maturity. These findings have had important implications, as indomethacin and other NSAIDs are now used for HO prophylaxis.

A limitation of our study is the retrospective study design, which does not allow for causality to be inferred. A further limitation could be that creatine phosphokinase levels were not analyzed in our study, although Sing et al. ${ }^{7}$ and Sherman et al. ${ }^{8}$ concluded a correlation of elevated serum levels of creatine phosphokinase in predicting $\mathrm{HO}$ in SCI patients. The correlation of serum creatine phosphokinase levels in predicting $\mathrm{HO}$ needs further investigation in prospective studies with higher patient numbers. So far, routine ultrasound examination remains the method of choice for early $\mathrm{HO}$ detection. ${ }^{15-17}$

In conclusion, the treatment of HO may be difficult and unsuccessful if not initiated early during the course of $\mathrm{HO}$ progression. Therefore, early diagnosis and intervention is crucial to avoid further complications. In this context, our study results show that assessment of CRP, AP and BAP levels is not a reliable method for diagnosing early $\mathrm{HO}$ in SCI patients.

\section{DATA ARCHIVING}

There were no data to deposit.

\section{CONFLICT OF INTEREST}

The authors declare no conflict of interest.

1 Citak M, Suero EM, Backhaus M, Aach M, Godry H, Meindl R et al. Risk factors for heterotopic ossification in patients with spinal cord injury: a case-control study of 264 patients. Spine 2012; 37: 1953-1957.

2 Wittenberg RH, Peschke U, Botel U. Heterotopic ossification after spinal cord injury. Epidemiology and risk factors. J Bone Joint Surg Br 1992; 74: 215-218.

3 Larson JM, Michalski JP, Collacott EA, Eltorai D, McCombs CC, Madorsky JB. Increased prevalence of HLA-B27 in patients with ectopic ossification following traumatic spinal cord injury. Rheumat Rehabil 1981; 20: 193-197.

4 Garland DE, Alday B, Venos KG. Heterotopic ossification and HLA antigens. Arch Phys Med Rehabil 1984; 65: 531-532.

5 Schurch B, Capaul M, Vallotton MB, Rossier AB. Prostaglandin E2 measurements: their value in the early diagnosis of heterotopic ossification in spinal cord injury patients. Arch Phys Med Rehabil 1997; 78: 687-691.

6 Estrores IM, Harrington A, Banovac K. C-reactive protein and erythrocyte sedimentation rate in patients with heterotopic ossification after spinal cord injury. J Spinal Cord Med 2004; 27: 434-437.

7 Singh RS, Craig MC, Katholi CR, Jackson AB, Mountz JM. The predictive value of creatine phosphokinase and alkaline phosphatase in identification of heterotopic ossification in patients after spinal cord injury. Arch Phys Med Rehabil 2003; 84: 1584-1588.

8 Sherman AL, Williams J, Patrick L, Banovac K. The value of serum creatine kinase in early diagnosis of heterotopic ossification. J Spinal Cord Med 2003; 26: 227-230.

9 Kress BC, Mizrahi IA, Armour KW, Marcus R, Emkey RD, Santora AC 2nd. Use of bone alkaline phosphatase to monitor alendronate therapy in individual postmenopausal osteoporotic women. Clin Chem 1999; 45: 1009-1017.

10 Kress BC, Mizrahi IA. Monitoring antiosteoporotic treatment of postmenopausal women using biochemical markers of bone turnover. Drugs Today (Barc) 1999; 35: 181-185.

11 Kress BC. Bone alkaline phosphatase: methods of quantitation and clinical utility. J Clin Ligand Assay 1998; 21: 139-148.

12 Maynard FM Jr, Bracken MB, Creasey G, Ditunno JF Jr, Donovan WH, Ducker TB et al. International standards for neurological and functional classification of spinal cord injury. American Spinal Injury Association. Spinal Cord 1997; 35: 266-274.

13 Orzel JA, Rudd TG. Heterotopic bone formation: clinical, laboratory, and imaging correlation. J Nucl Med 1985; 26: 125-132.

14 Kedlaya D, Nazir C. Heterotopic ossification with normal serum alkaline phosphatase levels: a case series. Arch Phys Med Rehabil 2005; 86: E44.

15 Bodley R, Jamous A, Short D. Ultrasound in the early diagnosis of heterotopic ossification in patients with spinal injuries. Paraplegia 1993; 31: 500-506.

16 Falsetti P, Acciai C, Lenzi L. Sonographic diagnosis of neurogenic heterotopic ossification in patients with severe acquired brain injury in a neurorehabilitation unit. J Clin Ultrasound 2011; 39: 12-17.

17 Falsetti P, Acciai C, Palilla R, Carpinteri F, Patrizio C, Lenzi L. Bedside ultrasound in early diagnosis of neurogenic heterotopic ossification in patients with acquired brain injury. Clin Neurol Neurosurg 2011; 113: 22-27. 